\title{
Intrathecal Trastuzumab Treatment in Patients with Breast Cancer and Leptomeningeal Carcinomatosis
}

\author{
Won-Young Park, MD ${ }^{1}$ \\ Han-Jo Kim, MD² \\ Kyoungha Kim, MD ${ }^{1}$ \\ Sang-Byung Bae, $\mathrm{MD}, \mathrm{PhD}^{2}$ \\ Namsu Lee, MD, PhD' \\ Kyu-Taek Lee, MD, PhD² \\ Jong-Ho Won, MD, PhD ${ }^{1}$ \\ Hee-Sook Park, MD, PhD ${ }^{1}$ \\ Sang-Cheol Lee, MD, PhD²
}

\begin{abstract}
Leptomeningeal carcinomatosis is a fatal manifestation of metastatic breast cancer. Investigation of intrathecal (IT) trastuzumab for leptomeningeal carcinomatosis is currently underway; however, there has been no consensus. We report on two cases of human epidermal growth factor receptor 2 positive (HER2+) breast cancer following IT trastuzumab for leptomeningeal carcinomatosis. The first patient was treated with weekly IT $15 \mathrm{mg}$ methotrexate plus IT $50 \mathrm{mg}$ trastuzumab for 7 months, followed by IT trastuzumab $(50 \mathrm{mg}>25 \mathrm{mg}$ ) for 18 months. The other patient received IT trastuzumab with systemic chemotherapy (trastuzumab and/or paclitaxel) for 13 months. Good control of leptomeningeal disease was achieved with IT trastuzumab in both patients, with survival durations of 20 and 29 months, respectively. We suggest that IT trastuzumab is a promising treatment for patients with HER2+ breast cancer and leptomeningeal carcinomatosis.
\end{abstract}

${ }^{1}$ Division of Hematology-Oncology, Department of Internal Medicine, Soonchunhyang University Hospital Seoul, Seoul, ${ }^{2}$ Division of Hematology-Oncology, Department of Internal Medicine, Soonchunhyang University Hospital Cheonan, Cheonan, Korea

Correspondence: Sang-Cheol Lee, MD, PhD Division of Hematology-Oncology, Department of Internal Medicine, Soonchunhyang University Hospital Cheonan, 31 Suncheonhyang 6-gil, Dongnam-gu, Cheonan 31151 , Korea

Tel: 82-41-570-3803

Fax: 82-41-574-5764

E-mail: sclee@schmc.ac.kr

Received August 8, 2014

Accepted November 14, 2014

Published Online March 2, 2015

\author{
Key words \\ Leptomeningeal carcinomatosis, Trastuzumab, \\ Intrathecal injection
}

\section{Introduction}

Leptomeningeal carcinomatosis is a rare but fatal manifestation of metastatic breast cancer, with limited treatment options [1]. Median overall survival of untreated patients with leptomeningeal carcinomatosis is only 4-6 weeks. Although the prognosis for patients with breast cancer and leptomeningeal carcinomatosis is relatively good compared with that of patients with other solid tumors, overall survival is 3-5 months [1,2]. Investigation of intrathecal (IT) treatment with trastuzumab for leptomeningeal carcinomatosis is currently underway; however, there has been no consensus [3]. We report on two cases of human epidermal growth factor receptor 2 positive (HER2+) breast cancer treated with IT trastuzumab for leptomeningeal carcinomatosis. 


\section{Case Report}

\section{Case 1}

A 47-year-old woman presented with a palpable mass on the left breast in May 2003. A modified radical mastectomy was performed, and HER2+ infiltrating ductal carcinoma (stage IIIB, nuclear grade I, estrogen receptor [ER]+, progesterone receptor $[\mathrm{PR}]+, \mathrm{HER} 2+$ [immunohistochemistry $2+$ and fluorescence in situ hybridization+]) was confirmed by histopathological examination. Metastasis to nine of 10 lymph nodes was detected, thus six cycles of adjuvant chemotherapy with cyclophosphamide, doxorubicin, and 5-fluorouracil (5-FU) were given, followed by adjuvant radiation therapy and hormonal therapy with toremifene.

In February 2005, she developed symptoms of central nervous system (CNS) involvement, including headache, nausea, and vomiting. Contrast-enhanced magnetic resonance imaging (MRI) of the brain showed multiple metastatic tumor masses in the cerebrum and cerebellum. She received whole brain radiotherapy for the brain metastases and started palliative hormone therapy with goserelin plus anastrozole.

Despite improvement in some brain metastatic lesions with therapy, in April 2007, contrast-enhanced brain MRI showed new brain metastases. Examination of the cerebrospinal fluid (CSF) showed a few degenerated lymphocytes. The treatment was changed to goserelin plus tamoxifen and was continued until February 2010 when metastases to the right supraclavicular lymph node, right neck, and chest wall were noted at restaging. After she underwent navigation-guided tumor mass removal of brain metastases and excision of the right chest wall mass in April 2010, she received three cycles of chemotherapy with docetaxel plus doxorubidin. She was subsequently treated with four additional palliative lines of chemotherapy, including 14 cycles of intravenous (IV) trastuzumab (July 2010-April 2011), xeloda plus lapatinib (May 2011-December 2011), gemcitabine plus cisplatin (February 2012, 1 cycle), and CMF (cyclophosphamide, methotrexate plus 5-FU, March 2012May 2012, 3 cycles). She underwent stereotatic radiosurgery (SRS) twice for brain metastases in 2008 and 2011.

In April 2012, the patient developed headache, vomiting, and gait disturbance. Newly visible and increased multifocal enhancing nodules in both frontal, the right parietal, and both cerebellar hemispheres were detected by contrastenhanced brain MRI (Fig. 1A). A CSF analysis showed many atypical cells. Metastases to the liver, common bile duct, lung, and right pleura were also confirmed by positron emission tomography-computed tomography.

After SRS for parenchymal metastases of the brain, weekly IT $50 \mathrm{mg}$ trastuzumab plus IT 15-mg methotrexate through the Ommaya reservoir was begun for the leptomeningeal nodules. Although she presented with mild weakness of the lower extremities, brain MRI showed remarkable improvement in the leptomeningeal nodules after 5 months of IT chemotherapy (Fig. 1B). In addition, CSF cytology was free of malignant or atypical cells during IT therapy, with complete resolution of symptoms in 2 days.

The therapy was well tolerated, and she received 20 administrations of weekly IT trastuzumab plus methotrexate
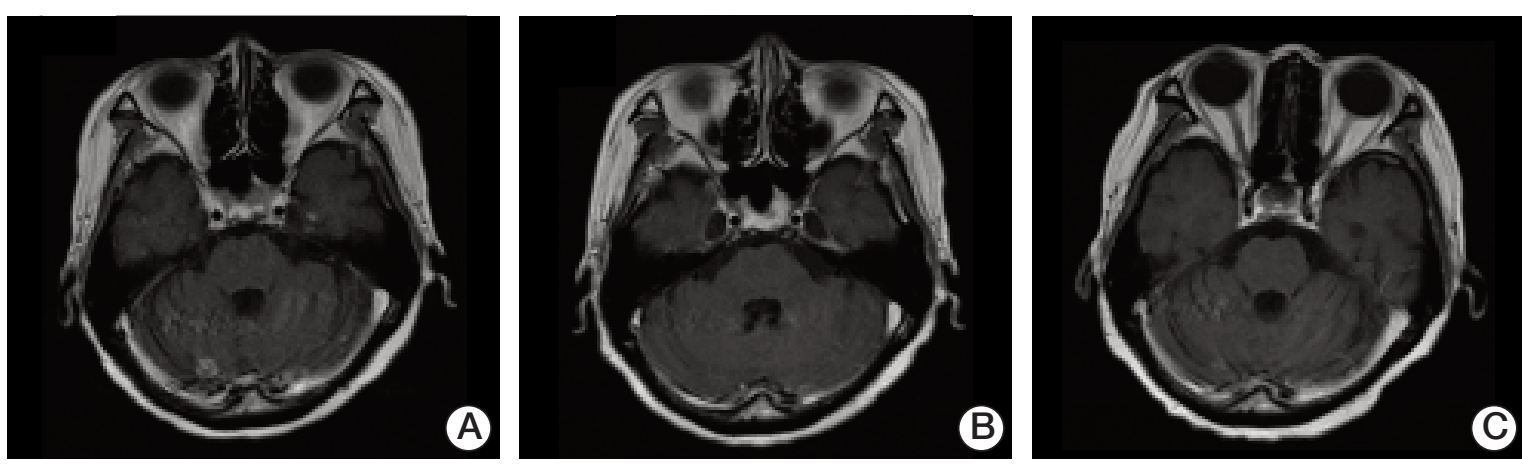

Fig. 1. Case 1 contrast-enhanced T1-weighted brain magnetic resonance image (MRI). (A) Multifocal enhanced nodules were detected in both frontal, the right parietal, and both cerebellar hemispheres at the beginning of intrathecal (IT) trastuzumab in April 2012. (B) A remarkable improvement in the leptomeningeal nodules was detected after 5 months of IT trastuzumab therapy in September 2012. (C) No evidence of newly developed enhancing nodules was detected in January 2013 after more than 9 months of IT trastuzumab therapy. Brain MRI shows complete remission of the brain mass (by Response Evaluation Criteria in Solid Tumors). 

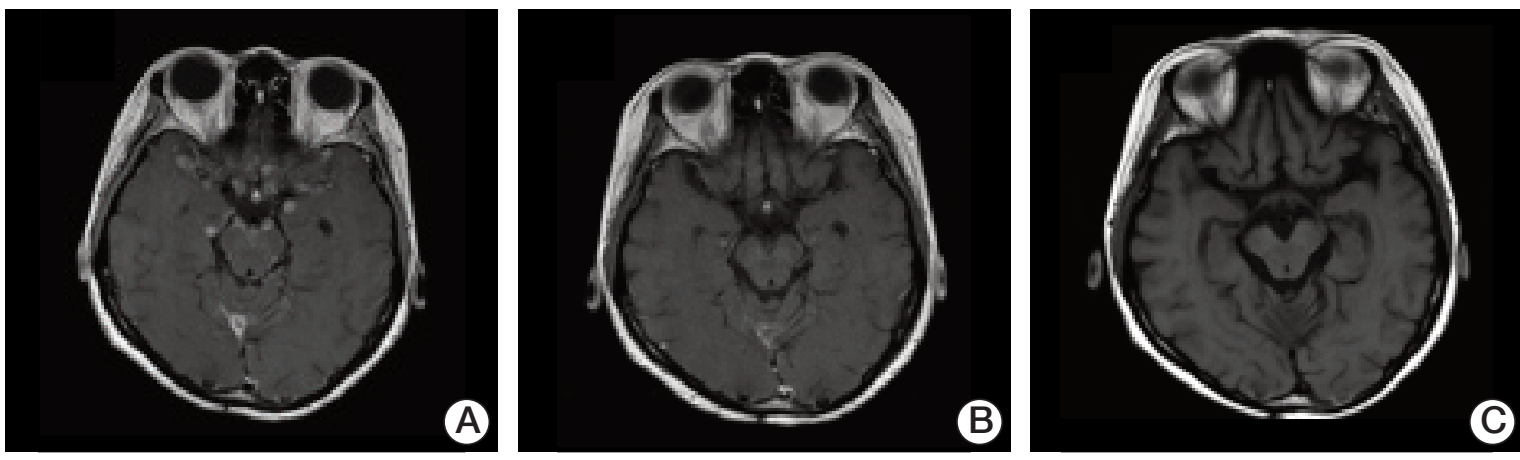

Fig. 2. Case 2 contrast-enhanced T1-weighted brain magnetic resonance image (MRI). (A) Contrast-enhanced MRI of the brain showed new multifocal leptomeningeal seeding in both Sylvian fissures, the prepontine cistern, the perimesencephalon, and the left cerebellopontine angle at the beginning of intrathecal (IT) trastuzumab treatment in January 2012. (B) Decreased size of the brain parenchymal metastatic mass and leptomeningeal nodules was documented after 8 months of IT trastuzumab therapy in September 2012, compared with brain MRI at the beginning of IT trastuzumab. (C) No evidence of newly developed enhancing nodules was detected after 18 months of IT trastuzumab therapy in July 2013.

over 7 months. The IT methotrexate was stopped transiently due to gait disturbance, but no evidence of newly developed enhancing nodules was observed on brain MRI until January 2013 (Fig. 1C). She continued weekly IT chemotherapy with a reduced dose of trastuzumab $(25 \mathrm{mg})$ for maintenance therapy and received 59 administrations during 18 months, with complete response of the brain mass, indicating complete disappearance of the radiological findings compared to the baseline examination [4].

Unfortunately, in November 2013, she refused further treatment due to progression of systemic disease and poor performance status. She died 2 months after the last IT trastuzumab administration in January 2014, after surviving more than 20 months since the diagnosis of leptomeningeal carcinomatosis.

\section{Case 2}

A 42-year-old woman presented with a palpable mass on the right breast in July 2008. Histopathological examination of the specimens sampled by fine-needle biopsy revealed HER2 amplified infiltrating ductal carcinoma (nuclear grade II, intermediate, ER-, PR-, HER2 3+; clinical stage cT2N1M0, IIB). After receiving four cycles of neoadjuvant chemotherapy with doxorubicin and docetaxel, she underwent a right half mastectomy with reconstruction in November 2008. Metastasis to five of 11 axillary lymph nodes was confirmed, thus, two cycles of doxorubicin and docetaxel were administered, followed by adjuvant radiation therapy.

In April 2010, she presented with headache, dizziness, nausea, dysarthria, and showed a tendency to fall to the right.
Contrast-enhanced brain MRI showed a metastatic tumor mass on the cerebellar vermis, and local relapse was also detected in the right breast by restaging. The intratentorial supracerebellar tumor mass was removed, and a duroplasty was performed with a modified radical mastectomy in May 2010. Definite improvement in neurological symptoms was observed after the surgery. She received xeloda single chemotherapy from July 2010 to March 2011.

However, she presented again in January 2012 with symptoms of increased intracranial pressure, including headache, nausea, and vomiting. Contrast-enhanced MRI of the brain showed new multifocal leptomeningeal seeding (Fig. 2A), and CSF obtained by lumbar puncture showed a few atypical cells and a slightly elevated protein level $(58.9 \mathrm{mg} / \mathrm{dL})$. She started weekly IT therapy with escalating doses of trastuzumab $(25 \mathrm{mg}>50 \mathrm{mg}$ ) with IV trastuzumab (2 mg/ $\mathrm{kg})$ plus IV paclitaxel $\left(80 \mathrm{mg} / \mathrm{m}^{2}\right)$. After eight cycles of chemotherapy, she was treated by whole brain radiotherapy, followed by weekly IV and IT trastuzumab $(50 \mathrm{mg}$ ) as maintenance therapy. In May 2012, after 16 cycles of therapy, her regimen was changed to three weekly IV trastuzumab plus IT trastuzumab (50 $\mathrm{mg}$ ) treatments.

The treatments were well tolerated without severe toxicity. The CSF remained free of malignant or even atypical cells during IT therapy, and, compared with the brain MRI at the beginning of the IT trastuzumab cycle, the brain parenchymal metastatic mass and leptomeningeal nodules had decreased in size (Fig. 2B and C).

The patient received a total of 33 administrations of weekly and three weekly IV plus IT trastuzumab treatments over 13 months with complete resolution of neurological symptoms 
(24 and 9 cycles, respectively). Although IT trastuzumab therapy was stopped due to dizziness, she remains alive, with survival of more than 29 months since being diagnosed with leptomeningeal carcinomatosis.

\section{Discussion}

Leptomeningeal carcinomatosis from HER2+ breast cancer is rare but important because of its increasing incidence and devastating clinical course [5]. Advances in early diagnosis of CNS diseases have been made in recent decades, and systemic chemotherapy, particularly with trastuzumab, has extended survival of patients with HER2+ breast cancer [6], however, despite aggressive treatment, prognosis of leptomeningeal carcinomatosis is still poor $[1,2]$.

The blood brain barrier (BBB) limits penetration of $>$ 200-kDa-sized molecules into the CNS [7,8]. Thus, systemically administered trastuzumab, a $185-\mathrm{kDa}$ monoclonal antibody, has only minimal infiltration into the CSF through an intact BBB. Pestalozzi and Brignoli [7] reported that the trastuzumab level in CSF was 300-fold lower than that in serum of patients with leptomeningeal carcinomatosis receiving IV weekly trastuzumab therapy. A study by Stemmler et al. [8] also showed that the ratio of median serum trastuzumab level to median CSF trastuzumab level was 420:1, although the ratio improved to $76: 1$ due to an impaired BBB after radiotherapy. Therefore, IT therapy with trastuzumab is an attractive therapeutic option for achievement of a high CSF drug concentration with a smaller dose, while reducing systemic drug toxicity.

In case studies reporting successful results of IT therapy with trastuzumab in patients with leptomeningeal carcinomatosis from HER2+ breast cancer, IT trastuzumab was administered in various doses of $25-150 \mathrm{mg}$ and using different schedules, including weekly to every 3 weeks $[4,9,10]$.
The case report by Hofer et al. [11] demonstrated the efficacy of IT trastuzumab at a dose of $150 \mathrm{mg}$ at 3 weekly intervals, but there is the question of whether long-term therapy over 6 months with such a high dose is safe and well-tolerable. According to a systematic review and pooled analysis by Zagouri et al. [4], a significant improvement in overall survival was observed in $68.8 \%$ of cases, but no evidence has shown that a particular method is superior to another.

Good control of leptomeningeal disease was achieved with IT trastuzumab in both of our patients, even though the first patient was heavily treated with five lines of cytotoxic chemotherapy and three lines of hormonal therapy, with survival durations of 20 and 29 months, respectively. Our report also demonstrates that long-term IT therapy with relatively high trastuzumab doses $(25-50 \mathrm{mg})$ for more than 1 year is safe and efficacious without severe disability of cognitive function.

We suggest that IT trastuzumab is a promising treatment for patients with HER2+ breast cancer and leptomeningeal carcinomatosis. Further studies are warranted in order to optimize dose, interval, maintenance duration, and combination drugs for treatment, and to standardize consensual response criteria.

\section{Conflicts of Interest}

Conflict of interest relevant to this article was not reported.

\section{Acknowledgments}

This work was supported by the Soonchunhyang University Research Fund.

\section{References}

1. Grossman SA, Krabak MJ. Leptomeningeal carcinomatosis. Cancer Treat Rev. 1999;25:103-19.

2. Jayson GC, Howell A. Carcinomatous meningitis in solid tumours. Ann Oncol. 1996;7:773-86.

3. Pienkowski T, Zielinski CC. Trastuzumab treatment in patients with breast cancer and metastatic CNS disease. Ann Oncol. 2010;21:917-24.

4. Zagouri F, Sergentanis TN, Bartsch R, Berghoff AS, Chrysikos
D, de Azambuja E, et al. Intrathecal administration of trastuzumab for the treatment of meningeal carcinomatosis in HER2positive metastatic breast cancer: a systematic review and pooled analysis. Breast Cancer Res Treat. 2013;139:13-22.

5. Clarke JL, Perez HR, Jacks LM, Panageas KS, Deangelis LM. Leptomeningeal metastases in the MRI era. Neurology. 2010; 74:1449-54.

6. Baselga J, Perez EA, Pienkowski T, Bell R. Adjuvant trastuz- 
umab: a milestone in the treatment of HER-2-positive early breast cancer. Oncologist. 2006;11 Suppl 1:4-12.

7. Pestalozzi BC, Brignoli S. Trastuzumab in CSF. J Clin Oncol. 2000;18:2349-51.

8. Stemmler HJ, Schmitt M, Willems A, Bernhard H, Harbeck N, Heinemann V. Ratio of trastuzumab levels in serum and cerebrospinal fluid is altered in HER2-positive breast cancer patients with brain metastases and impairment of blood-brain barrier. Anticancer Drugs. 2007;18:23-8.

9. Le Rhun E, Taillibert S, Chamberlain MC. Carcinomatous meningitis: Leptomeningeal metastases in solid tumors. Surg Neurol Int. 2013;4(Suppl 4):S265-88.

10. Mir O, Ropert S, Alexandre J, Lemare F, Goldwasser F. Highdose intrathecal trastuzumab for leptomeningeal metastases secondary to HER-2 overexpressing breast cancer. Ann Oncol. 2008;19:1978-80

11. Hofer S, Mengele K, Stemmler HJ, Schmitt M, Pestalozzi B. Intrathecal trastuzumab: dose matters. Acta Oncol. 2012;51: 955-6. 